\title{
Distúrbios endocrinológicos na Distrofia Muscular de Duchenne: Um relato de caso
}

\author{
Endocrinological disorders in Duchenne Muscular Dystrophy: A case report \\ Trastornos endocrinológicos en la Distrofia Muscular de Duchenne: Reporte de um caso
}

Recebido: 01/07/2021 | Revisado: 06/07/2021 | Aceito: 07/07/2021 | Publicado: 17/07/2021

\author{
Gabriela Sacuno Bonilha \\ ORCID: https://orcid.org/0000-0002-1689-6126 \\ Centro Universitário da Fundação Assis Gurgacz, Brasil \\ E-mail: gabrielasacuno@hotmail.com \\ Isabel Cristina Ranzan \\ ORCID: https://orcid.org/0000-0002-4940-8184 \\ Centro Universitário da Fundação Assis Gurgacz, Brasil \\ E-mail: isabelranzan@ hotmail.com \\ Marise Vilas Boas Pescador \\ ORCID: https://orcid.org/0000-0003-3718-1063 \\ Centro Universitário da Fundação Assis Gurgacz, Brasil \\ E-mail: marisevilasboas@ hotmail.com
}

\begin{abstract}
Resumo
A Distrofia Muscular de Duchenne (DMD) é uma doença hereditária, autossômica recessiva ligada ao X, acometendo, principalmente, o sexo masculino. Há o comprometimento muscular progressivo, começando pelos músculos proximais dos membros inferiores, depois ocorre redução da força muscular dos membros superiores e comprometimento dos músculos cardíacos e respiratórios, não existindo terapia curativa. Dessa forma, este trabalho tem como objetivo relatar um caso de um paciente com distrofia muscular de Duchenne, enfatizando os possíveis efeitos endocrinológicos que podem manifestar-se nessa patologia. A melhor medicação para o manejo da doença é o corticosteroide, o qual melhora a força muscular, prologando o tempo de marcha, preservando a função dos membros superiores, prevenindo escoliose, reduzindo progressão da cardiomiopatia e atrasando a necessidade de ventilação invasiva. No entanto, essa medicação oferece uma gama de efeitos adversos a esses pacientes, se fazendo necessário o atendimento endocrinológico, com os seguintes objetivos: monitorar o crescimento, desenvolvimento puberal, diagnosticar deficiências hormonais, fornecendo terapia de reposição quando necessário e prevenindo a ocorrência de crise adrenal.
\end{abstract}

Palavras-chave: Distrofias musculares; Distrofia muscular de Duchenne; Endocrinologia.

\begin{abstract}
Duchenne Muscular Dystrophy (DMD) is an inherited, autosomal recessive X-linked disease, affecting mainly males. There is progressive muscle impairment, starting with the proximal muscles of lower limbs, then reduction of upper limbs muscle strength and impairment of cardiac and respiratory muscles, with no curative therapy. Thus, this is a case report of a patient with Duchenne Muscular Dystrophy, emphasizing the possible endocrinological effects that can manifest in this pathology. The best medication for the management of Duchenne Muscular Dystrophy is corticosteroid, which improves muscle strength, extending gait time, preserving the function of upper limbs, preventing scoliosis, reducing cardiomyopathy progression and delaying invasive ventilation need. However, this medication offers a range of adverse effects to these patients, making endocrinological care necessary, with the following objectives: monitoring growth, pubertal development, diagnosing hormonal deficiencies, providing replacement therapy when necessary and preventing the occurrence of adrenal crisis.
\end{abstract}

Keywords: Muscular dystrophy; Duchenne muscular Dystrophy; Endocrinology.

\section{Resumen}

La distrofia muscular de Duchenne (DMD) es una enfermedad hereditaria autosómica recesiva ligada al cromosoma $\mathrm{X}$, que afecta principalmente a los hombres. Hay un deterioro muscular progresivo, comenzando por los músculos proximales de los miembros inferiores, luego hay una reducción de la fuerza muscular en los miembros superiores y un deterioro de los músculos cardíaco y respiratorio, sin tratamiento curativo. Así, este trabajo tiene como objetivo reportar un caso de un paciente con distrofia muscular de Duchenne, enfatizando los posibles efectos endocrinológicos que pueden manifestarse en esta patología. El mejor medicamento para controlar la enfermedad son los corticosteroides, que mejoran la fuerza muscular, prolongan el tiempo de caminata, preservan la función de las extremidades superiores, previenen escoliosis, reducen progresión de la miocardiopatía y retrasan la necesidad de ventilación invasiva. Sin embargo, este medicamento ofrece un abanico de efectos adversos a estos pacientes, 
requiriendo cuidados endocrinológicos, con los siguientes objetivos: monitorizar el crecimiento, desarrollo puberal, diagnosticar deficiencias hormonales, proporcionar terapia sustitutiva cuando sea necesario y prevenir la aparición de crisis suprarrenales.

Palabras clave: Distrofias musculares; Distrofia muscular de Duchenne; Endocrinología.

\section{Introdução}

A distrofia muscular de Duchenne (DMD) é uma doença hereditária com herança recessiva ligada ao X, que acomete os músculos de maneira progressiva, ocorrendo principalmente no sexo masculino. O comprometimento muscular ocorre primeiro nos músculos proximais dos membros inferiores, com redução da força muscular e contraturas progressivas, levando ao comprometimento da marcha. Posteriormente, há uma redução da força muscular dos membros superiores, em que os pacientes evoluem com contraturas nos braços e comprometimento dos músculos cardíacos e respiratórios (Araujo et al, 2018).

O tratamento da doença é de suporte, não havendo cura. O corticosteroide é o medicamento de escolha no manejo desses pacientes, retardando a progressão da doença (Annexstad et al, 2014). No entanto, o uso crônico dessa medicação implica uma série de efeitos colaterais que somado a implicações da própria Distrofia Muscular de Duchenne, exige acompanhamento com outros especialistas: cardiologista, pneumologista e endocrinologista. Relata-se um caso de um paciente com distrofia muscular de Duchenne, enfatizando os possíveis efeitos endocrinológicos que podem manifestar-se nessa patologia.

\section{Metodologia}

Trata-se de um estudo na modalidade relato de caso único, com abordagem qualitativa. As características de um relato de caso, de acordo com Parente et al (2010), são a descrição detalhada de características importantes sobre sinais, sintomas e outras características do paciente, relatando os procedimentos terapêuticos realizados e o desenvolvimento do caso. As informações necessárias para o desenvolvimento do relato foram obtidas por meio de prontuários e entrevistas com o paciente e familiares. $\mathrm{O}$ atendimento ocorreu em um centro de atenção secundária no município de Cascavel/PR. Foi utilizado como base metodológica o trabalho de Pereira et al (2018) e Yin (2015).

\section{Resultados}

Paciente do sexo masculino, 8 anos, encaminhado ao neurologista com queixas de dificuldade na alfabetização e concentração, alterações no comportamento, dificuldade na coordenação motora ampla e fina, dificuldade para correr, apresentando dor em membros inferiores e quadril. Além disso, mãe relata quedas frequentes e com facilidade há 1 ano. História gestacional: gestação e pré-natal sem anormalidades, nascido por parto cesáreo por bolsa rota com 38 semanas de idade gestacional. Peso ao nascer: 3,265g e talhe de $47 \mathrm{~cm}$. Ao exame físico, observou-se hiperatividade durante toda a consulta, força reduzida em cintura pélvica, sinal de Gowers, hipotrofia muscular proximal e hipertrofia de panturrilhas. Os exames laboratoriais solicitados confirmaram a hipótese diagnóstica de miopatia (Tabela 1), os testes sorológicos foram não reagentes e a tomografia computadorizada de crânio normal. O diagnóstico de Distrofia Muscular de Duchenne foi confirmado em estudo genético, pela identificação do gene DMD (Distrofina, OMIM* 300377) em hemizigose. 
Tabela 1: Exames laboratoriais iniciais.

\begin{tabular}{lcc}
\hline Exame & Resultado & $\begin{array}{c}\text { Valor de referência } \\
\text { (unidade) }\end{array}$ \\
\hline CPK & 6700 & 32 a $294 \mathrm{U} / \mathrm{L}$ \\
CKMB & 300 & Até $5,0 \mathrm{ng} / \mathrm{ml}$ \\
LDH & 788 & Até $300 \mathrm{U} / \mathrm{L}$ \\
TGO & 154 & Até $40 \mathrm{U} / \mathrm{L}$ \\
LACTATO & 1,1 & Até $2 \mathrm{mml} / \mathrm{L}$ \\
URÉIA & 28 & $2-34 \mathrm{mg} / \mathrm{dL}$ \\
CREATININA & 0,14 & 0,4 a $0,9 \mathrm{mg} / \mathrm{Dl}$ \\
FAN & Não reagente & Não reagente \\
ANTI- & Não reagente & Não reagente \\
ACETILCOLINA & & \\
\hline
\end{tabular}

Fonte: Autores (2021).

Foi iniciado acompanhamento em ambulatório especializado em doenças neuromusculares e prescrito tratamento com Prednisolona $15 \mathrm{mg}$ ao dia em tomada única pela manhã. Sendo solicitado avaliação especializada com cardiologista, endocrinologista e pneumologista. O eletrocardiograma e espirometria não apresentaram alterações, mantendo acompanhamento periódico com cardiologista e pneumologista.

A avaliação com endocrinologista pediátrico foi realizada aos 7 anos e 10 meses de idade, sem queixas específicas no momento da consulta. A estatura alvo familiar era de 169,5cm (-0,96 DP; variando de 0,13 a -2,0 DP), ao exame físico o menor apresentava estatura de $113 \mathrm{~cm}$ (-2,44 DP) e peso em $19 \mathrm{~kg}$ (-2,12 DP), sendo o índice de massa corporal (IMC) adequado para a idade e sexo (-0,59 DP), o gráfico 1 demonstra o escore $\mathrm{Z}$ da estatura e sua relação com a estatura alvo familiar. O estadiamento de Tanner para avaliação do desenvolvimento puberal demonstrou que o menor se apresentava impúbere. A avaliação laboratorial realizada estava normal (Tabela 2), apresentando apenas deficiência de vitamina D (17,8 ng/mL, normal > 20 para a idade). A radiografia de mãos e punhos para avaliação da maturidade óssea foi de 7 anos, com a previsão de estatura final em $162 \mathrm{~cm}$ (limite inferior da estatura alvo). Como conduta terapêutica foi iniciado reposição com Colecalciferol 14000 UI por semana e optado por reavaliação periódica para acompanhamento da velocidade de crescimento e da evolução do crescimento e desenvolvimento puberal. 
Research, Society and Development, v. 10, n. 8, e54910817773, 2021

(CC BY 4.0) | ISSN 2525-3409 | DOI: http://dx.doi.org/10.33448/rsd-v10i8.17773

Gráfico 1. Estatura para idade e sexo comparativo com o canal alvo familiar.

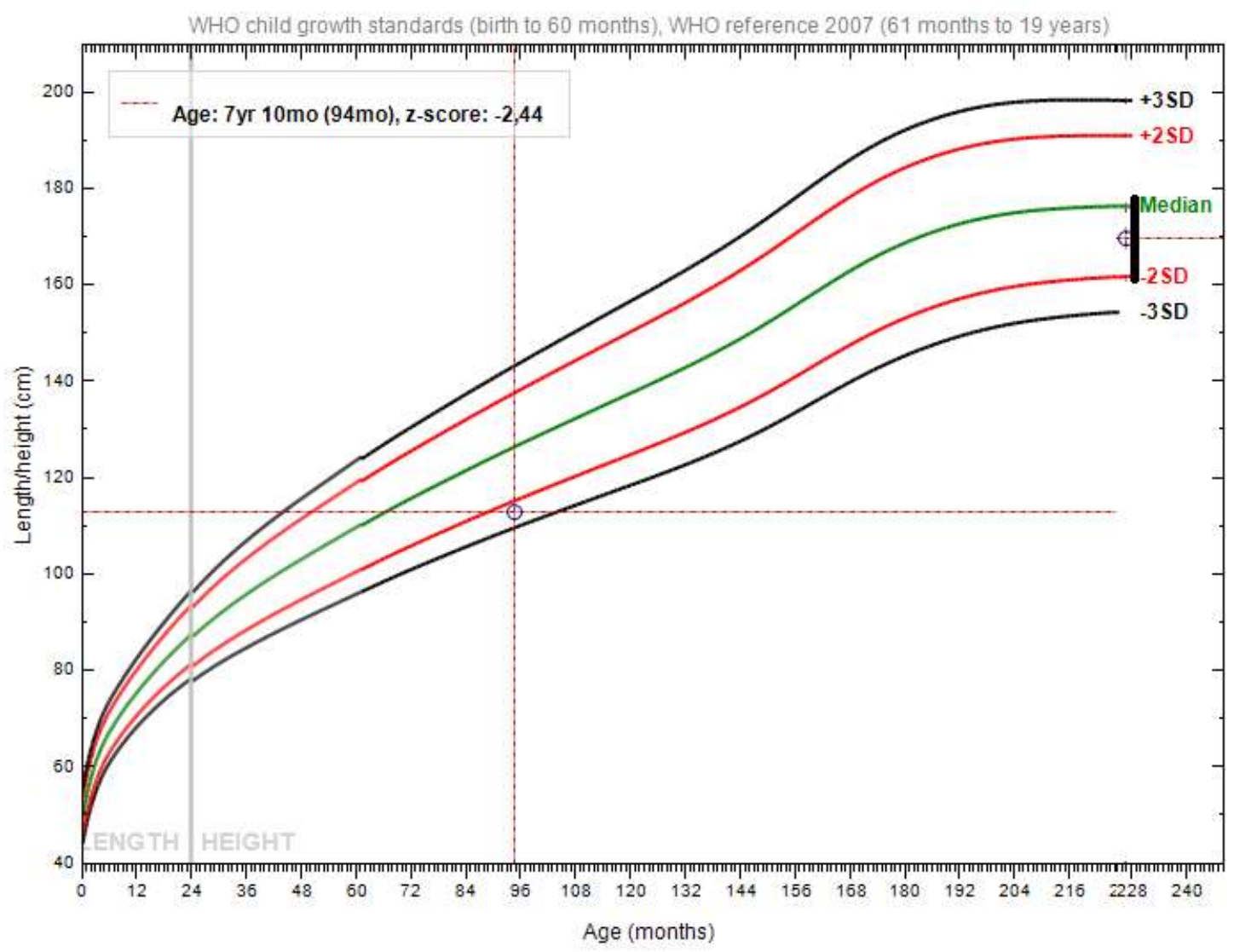

Fonte: Autores (2021).

Tabela 2: Exames laboratoriais solicitados em avaliação endocrinológica.

\begin{tabular}{lcc}
\hline Exame & Resultado & Valor de referência \\
\hline TSH & 3,04 & 0,3 a $4,2 \mu \mathrm{UI} / \mathrm{mL}$ \\
Cálcio & 9,1 & 8,6 a $10 \mathrm{mg} / \mathrm{dL}$ \\
Fósforo & 5,3 & 4,0 a $7,0 \mathrm{mg} / \mathrm{dL}$ \\
Fosfatase alcalina & 124 & $\leq 350 \mathrm{U} / \mathrm{L}$ \\
25 Hidroxi vitamina & 17,8 & $>20 \mathrm{ng} / \mathrm{mL}$ \\
D & & \\
PTH & 25 & 15 a $65 \mathrm{pg} / \mathrm{mL}$ \\
IGF-1 & 142 & 68 a $316 \mathrm{ng} / \mathrm{mL}$ \\
\hline
\end{tabular}

Fonte: Autores (2021).

\section{Discussão}

A distrofia muscular de Duchenne (DMD) é uma doença hereditária com herança recessiva ligada ao X, que afeta os músculos de forma progressiva, ocorrendo principalmente no sexo masculino. O comprometimento muscular ocorre primeiro nos músculos proximais dos membros inferiores, com redução da força muscular e contraturas progressivas, levando ao 
Research, Society and Development, v. 10, n. 8, e54910817773, 2021

(CC BY 4.0) | ISSN 2525-3409 | DOI: http://dx.doi.org/10.33448/rsd-v10i8.17773

comprometimento da marcha. Posteriormente, há uma redução da força muscular dos membros superiores, em que os pacientes evoluem com contraturas nos braços e comprometimento dos músculos cardíacos e respiratórios. Apesar de não existir uma terapia curativa, atualmente, a maioria dos pacientes caminham até os 13 anos de idade, alguns até os 20 anos, e alguns sobrevivem por mais de 30 anos com o auxílio de suporte ventilatório e cardíaco. O defeito na proteína chamada distrofina, que se liga às proteínas F-actina e sarcolema, é a responsável pela doença, não permitindo que haja flexibilidade da membrana plasmática durante a contração muscular, não havendo a manutenção da estrutura da fibra. Com as novas técnicas de biologia molecular, o diagnóstico de DMD tornou-se mais fácil, dispensando-se a realização de biópsia muscular (Werneck et al, 2019; Yiu \& Kornberg, 2015; Hoffman et al, 1987; Kunkel et al,1986).

A incidência da DMD é de aproximadamente 1 caso para cada 3.500 a 5.000 meninos nascidos vivos, podendo estar associada ou não a retardo mental. São importantes o diagnóstico precoce e o entendimento de que não há medicamento capaz de parar completamente o avanço das deficiências funcionais. Por isso, um bom manejo das complicações tem modificado a expectativa de vida desses pacientes, visto que sua mortalidade está relacionada as complicações cardíacas e pulmonares (Araujo et al, 2018; Moat et al, 2013; Suthar \& Sankhyan, 2017).

Em 1974, a Prednisona foi usada empiricamente no tratamento da DMD, sendo observado uma melhora na mobilidade, marcha e agilidade, bem como uma redução sérica da creatina quinase. Dessa forma, nesse estudo, concluiu-se que a Prednisona melhorava a deambulação e a qualidade de vida dos pacientes acometidos. Outro estudo realizado em 1989, constatou a melhora nas atividades funcionais em pacientes em uso de Prednisona por um período de 6 meses. Assim, a Prednisona passou a ser prescrita de forma rotineira, sendo os seus benefícios maiores do que os efeitos colaterais. Outros estudos também confirmaram a eficácia dessa medicação, observando uma melhora da função muscular de membros superiores e inferiores, preservando a força por um tempo maior, melhorando a qualidade de vida e sobrevivência, visto que tem efeitos benéficos na ventilação pulmonar e cardiomiopatia. Além disso, observou-se uma redução no número de cirurgias de escoliose, ajudando na sua prevenção; atraso na necessidade de ventilação invasiva; levando ao prolongamento da expectativa de vida para 30 anos (Werneck et al, 2019; Bushby et al, 2010).

No entanto, o uso de corticosteroides está associado a efeitos adversos significativos, como atraso na puberdade, baixa estatura, obesidade, osteoporose, alterações cutâneas, aparência cushingoide, distúrbios comportamentais, supressão adrenal, catarata e alterações metabólicas (Bianchi et al, 2011b).

\subsection{Crescimento}

Apesar do peso e o comprimento serem normais ao nascimento, há um retardo gradual no crescimento ao longo do primeiro ano de vida desses pacientes. Aos 10 anos, o comprimento médio cai para o percentil 50; aos 18 anos, muitos dos pacientes caem para o percentil 5 de altura (Leung et al, 2011). Em resumo, foi observado: comprimento normal ao nascer, velocidade abaixo da média no início da vida, velocidade de crescimento adequada para a idade com altura no percentil abaixo da média no decorrer da infância (Weber et al, 2018a).

A etiologia do retardo no crescimento na DMD é desconhecida, visto que a secreção de hormônio de crescimento (HC) em resposta ao teste provocativo, a circulação de fatores de crescimento e maturação do esqueleto são normais nessa população (Weber et al, 2018a).

Além disso, a corticoterapia exacerba esse retardo no crescimento, visto que há uma inibição do HC, antagonizando a ação dele e do fator de crescimento semelhante a insulina - IGF-1, induzindo apoptose de condrócitos na placa de crescimento (Bianchi et al, 2011b). Assim, o crescimento é lento 12 a 18 meses depois do início do tratamento com corticosteroides. A 
baixa estatura ajuda na preservação da função muscular, caminhada e subida de escadas, mas ainda é uma preocupação grande entre os pacientes e familiares (Weber et al, 2018a; Ward e Weber, 2019).

A baixa estatura secundária ao uso crônico de corticosteroides pode ter um impacto negativo na saúde psicossocial, qualidade de vida e resultado clínico. A velocidade de crescimento e o HC são normais na ausência de tratamento com corticoide. Os corticosteroides fazem supressão da produção de HC e/ou resistência ao HC ou ao IGF-1, bem como efeitos diretos nos ossos (Bianchi et al, 2011b).

O crescimento deve ser monitorado a cada 6 meses até o final da puberdade e até que alcance a estatura final. Quando houver crescimento prejudicado, ou seja, percentil para altura decrescendo, velocidade de crescimento $<4 \mathrm{~cm} /$ ano ou altura abaixo do terceiro percentil, deve-se solicitar consulta e acompanhamento com endocrinologista (Weber et al, 2018a).

O tratamento da baixa estatura nessa população é controverso, pois acredita-se que o aumento do crescimento pode piorar a função muscular. Assim, a indicação de tratamento deve ser individualizada, avaliando-se o custo-benefício para cada paciente, não sendo regularmente recomendado o uso de hormônio de crescimento recombinante. O uso deste deve ser realizado quando há evidencias bioquímicas de deficiência de hormônio hipotalâmico estimulador da secreção de hormônio de crescimento (GHRH) e/ou de hormônio de crescimento hipofisário, com discussão dos riscos conhecidos e ainda desconhecidos associados a essa terapia (Weber et al, 2018a).

Um estudo retrospectivo obteve sucesso apenas a curto prazo na terapia com HC recombinante. Após algum tempo, os pacientes que participaram do estudo apresentaram efeitos colaterais como hipertensão intracraniana, intolerância à glicose e progressão da escoliose (Weber et al, 2018a).

$\mathrm{O}$ uso do IGF-1 é aprovado para o tratamento de pacientes com baixa estatura relacionado à resistência ao HC ou deficiência severa de IGF-1. Assim, é esperado que haja um aumento no crescimento e melhora na função muscular em pacientes com DMD, sem risco de resistência à insulina (Leung et al, 2011).

O manejo da baixa estatura deve ser individualizado, a fim de atender as necessidades do paciente e de seus familiares, podendo incluir: nenhuma intervenção; redução da dose de corticosteroides ou mudança para seu uso intermitente (na maioria das vezes é inviável); e administração de HC recombinante (Bianchi et al, 2011b).

\subsection{Puberdade}

O atraso puberal é multifatorial: genética, doença crônica e supressão da glândula pituitária por corticoide exógeno (Leung et al, 2011). O fator mais significativo será o excesso de corticosteroides, que inibe a produção de hormônios hipotalâmicos-hipofisários reguladores da puberdade, resultando em deficiência de testosterona devido ao hipogonadismo hipogonadotrófico (Bianchi et al, 2011b). Os efeitos do hipogonadismo podem incluir atraso na puberdade, diminuição ou retardo no crescimento, maturação emocional prejudicada, baixa autoestima, osteoporose e anormalidade metabólicas (Leung et al, 2011). Estudos revelaram que o atraso na puberdade é comum, com prevalência entre 50 e $100 \%$ em meninos em tratamento com glicorticoides (Weber et al, 2018a; Wood et al, 2015).

A avaliação inicial para o hipogonadismo inclui dosagem de nível sérico de testosterona pela manhã, teste de função tireoidiana e estimativa da idade óssea com radiografia de mão esquerda (Leung et al, 2011). Todos os pacientes com DMD devem fazer o monitoramento da puberdade a cada 6 meses, começando aos 9 anos de idade (Weber et al, 2018a).

O tratamento do atraso puberal pode envolver a reposição de testosterona em baixas doses por volta dos 14 anos de idade, quando o volume testicular for menor do que $4 \mathrm{~cm}^{3}$; ou com 12 anos, naqueles pacientes em tratamento com glicocorticoides (Bianchi et al, 2011b). Deve ser começado em baixas doses, com aumento gradual das doses por até 3-4 anos 
até serem atingidas doses de reposição esperadas para um adulto. As baixas doses de testosterona podem ser administradas mensalmente intramuscular ou com aplicação transdérmica diariamente (Weber et al, 2018a).

Em geral, a testosterona promove virilização e crescimento, com ganho de densidade óssea, força muscular e energia, havendo um aumento de massa magra e redução de massa gorda (Bianchi et al, 2011b). É importante aconselhar os pacientes e familiares sobre os demais efeitos desse tratamento que inclui odor corporal, pelos faciais, surto de crescimento, fechamento da placa de crescimento e aumento de libido (Weber et al, 2018a).

Os níveis de hormônio devem ser monitorados para o ajuste da dose e o acompanhamento anual dos níveis de hemoglobina e hematócrito, lipídios e glicose sérica. Em alguns casos, há efeito adverso no músculo e no coração, sendo motivo de interrupção do tratamento. Estudos maiores são necessários para o melhor estabelecimento de tempo e dose da terapia com testosterona (Weber et al, 2018a).

\subsection{Ganho de peso}

Os pacientes com DMD apresentam um risco maior de ganho de peso, resistência insulínica e Diabetes tipo 2, decorrente do uso crônico de corticosteroides e da fraqueza muscular progressiva. Além dos corticosteroides estimularem o apetite, eles agem na via metabólica hepática e nas células adiposas, promovendo resistência insulínica, aumento da glicemia e da adiposidade visceral. A fraqueza muscular progressiva leva a uma limitação na atividade física, o que predispõe ainda mais ao ganho de peso. O excesso de peso se torna prejudicial em diversos aspectos: pode levar a intolerância a carboidratos, diabetes, prejudicar as funções pulmonar e cardíaca. Além disso, o excesso de peso traz um ônus aos familiares e cuidadores na mobilidade desses meninos, principalmente, quando estes perdem a capacidade de deambulação (Bianchi et al, 2011b).

No entanto, há também um fator dietético associado à obesidade em pacientes com DMD, sendo constatado em estudos observacionais uma maior ingesta calórica por esses pacientes, mesmo antes do início da corticoterapia (Leung et al, 2011).

O acompanhamento do peso deve ser feito em todas as consultas, pesando o paciente, colocando na curva de crescimento e calculando o índice de massa corporal. O objetivo é manter o IMC dos pacientes entre os percentis 10 e 85 para a idade e sexo. A intervenção deve ser feita quando houver uma velocidade de ganho de peso em aumento exponencial (Leung et al, 2011). O monitoramento do peso deve ser realizado de forma mais criteriosa em pacientes com IMC e Z-score aumentados (IMC > percentil 85 e Z-score > 0,5). Nesses casos, deve ser feito um programa de manejo intensivo interdisciplinar, visando o aconselhamento e orientações de técnicas de mudança comportamental (Weber et al, 2018a).

A estratégia de prevenção da obesidade deve começar em três momentos: diagnóstico, início da corticoterapia e perda da mobilidade. As estratégias devem envolver dieta, duração adequada do sono, avaliação psicossocial e suporte ao paciente e cuidadores. O estímulo a atividade física ainda é controverso, mas se torna importante para evitar a atrofia por desuso e complicações por inatividade. Assim, deve-se orientar a realização de atividades leves que visam o fortalecimento funcional, com exercícios em piscina e de recreação (Weber et al, 2018a).

As complicações metabólicas da obesidade incluem desregulação da glicose, DM2, dislipidemias e hipertensão. Crianças e jovens com excesso de peso apresentam risco maior de isolamento social, estigmatização, distúrbios psiquiátricos (depressão, ansiedade), dificuldades escolares, insatisfação com o corpo, distúrbios alimentares, provocações e bullying. Os pacientes devem ser monitorados rotineiramente pela história clínica, visto que distúrbios da saúde mental e alguns agentes farmacoterapêuticos que são usados podem promover o ganho de peso e afetar o prognóstico (Weber et al, 2018a). 
Research, Society and Development, v. 10, n. 8, e54910817773, 2021

(CC BY 4.0) | ISSN 2525-3409 | DOI: http://dx.doi.org/10.33448/rsd-v10i8.17773

\subsection{Osteoporose}

As fraturas são problemas significativos nos pacientes com DMD, sendo causas subdiagnosticadas de dor, perda de mobilidade e deformidade esquelética. Por isso, logo após o diagnóstico da doença, deve-se obter informações básicas sobre a saúde óssea, incluindo detalhada história nutricional, história de fratura, confirmação radiológica de fraturas passadas e avaliação de dor na coluna. Os níveis séricos de vitamina $\mathrm{D}$ devem ser dosados e, quando necessário, prescrita, sendo o valor alvo de $30 \mathrm{ng} / \mathrm{ml}$ ou $75 \mathrm{nmol} / \mathrm{ml}$. Algumas publicações recomendam a suplementação de vitamina D quando os níveis estiverem abaixo de $20 \mathrm{ng} / \mathrm{ml}$ ou $50 \mathrm{nmol} / \mathrm{ml}$. Após o início do tratamento com corticoides, a dosagem de vitamina D pode ser feita a cada 1 ou 2 anos (Leung et al, 2011; Ward et al, 2018; Salera et al, 2017).

Foi estimado uma perda de 6 a $12 \%$ na densidade óssea dos pacientes no primeiro ano de terapia com corticosteroide e um aumento rápido no risco de fratura nos primeiros 3 meses de tratamento. Cerca de 30 a $50 \%$ dos pacientes adultos que fazem uso crônico de corticoides tem fraturas, sendo as fraturas vertebrais assintomáticas devido ao efeito analgésico dos corticoides. O tratamento da doença aumenta o risco de fraturas vertebrais e de membros inferiores. $32 \%$ dos pacientes em tratamento com corticoide apresentaram compressões vertebrais, enquanto foi observado uma incidência 2,6x maior de fraturas de ossos longos em pacientes em tratamento (Bianchi et al, 2011b).

Há estudos que avaliaram os benefícios da administração de cálcio e de vitamina D em pacientes com DMD. Os pacientes que foram suplementados e alcançaram níveis de ingestão de cálcio na dieta mostraram redução da reabsorção óssea, reduzindo os níveis hormonais da paratireoide e um aumento da densidade mineral óssea na coluna lombar e em todo o corpo. A suplementação deve ser considerada apenas em casos de ingestão alimentar inadequada, pois a ingestão de cálcio e vitamina D na alimentação se mostrou superior às formas suplementares. Outra recomendação é a limitação no consumo de sódio, a fim de reduzir a excreção de cálcio urinário, particularmente durante o tratamento com corticosteroides (Leung et al, 2011; Bianchi et al, 2011a).

Outras opções de tratamento da osteopenia e osteoporose estão sendo discutidos, incluindo bifosfonados, Teriparatida, paratormônio recombinante e indução da puberdade com testosterona. No entanto, o aumento do risco de osteossarcoma em pacientes pediátricos limita o uso da Teriparatida. Por esse motivo, os bifosfonados ainda são o tratamento padrão-ouro para prevenção e tratamento para osteoporose induzida por glicorticoides (Bianchi et al, 2011b). Alendronato e Risedronato são os mais utilizados, sendo a eficácia semelhante em pacientes do sexo feminino e masculino. O tratamento com bifosfonados intravenoso se mostrou eficaz em pacientes com fratura vertebral e dor nas costas (Leung et al, 2011).

Em 2010, foi realizado a Segunda Conferência dos Aspectos Endócrinos da Distrofia Muscular de Duchenne, na qual foram discutidos quatro tópicos principais: crescimento, puberdade e autoestima; problemas metabólicos e obesidade; saúde óssea; tratamento endocrinológico para a função muscular (Leung et al, 2011). As recomendações da Conferência forneceram uma estrutura de triagem e gerenciamento da saúde óssea e as complicações endócrinas da DMD, reconhecendo a grande falta de pesquisas baseadas em evidências nessa área. As recomendações envolvem: avaliação da densidade mineral óssea por absorciometria com raio X de dupla energia (DXA) a partir do diagnóstico da doença e/ou após início da corticoterapia, suplementação de cálcio e/ou vitamina D nos pacientes com deficiência, considerar uso de bifosfonados em pacientes com fraturas (Weber et al, 2018b).

Em 2014, houve uma atualização das diretrizes da Conferência de 2010, a fim de melhorar o manejo desses pacientes. Essa atualização foi direcionada a melhorar a avaliação e intervenção dos pacientes, devido ao aumento da taxa de sobrevida da população acometida. Assim, foram acrescentados três novos tópicos em relação aos cuidados originais: cuidados primários e gerenciamento de emergências, gerenciamento endócrino e transições de cuidados ao longo da vida (Birnkrant et al, 2018). 
Os objetivos do atendimento endócrino são monitorização do crescimento, do desenvolvimento puberal, bem como a realização do diagnóstico de deficiências hormonais, fornecendo terapia de reposição quando necessário e prevenção de crise adrenal, pois esta oferece risco de vida ao paciente (Birnkrant et al, 2018). No entanto, muitas coisas permanecem desconhecidas em relação à história natural do sistema endócrino na Distrofia Muscular de Duchenne dificultando o gerenciamento dessas questões na população acometida. Estudos observacionais são necessários para determinar a relação dos resultados com os sintomas endócrinos. Dosagens de níveis hormonais, marcadores de remodelação óssea e estudos de imagem óssea em diferentes estágios de desenvolvimento podem ser coletados para determinar a ação dos marcadores conforme o tempo (Leung et al, 2011).

\section{Considerações Finais}

A Distrofia Muscular de Duchenne é uma doença hereditária rara que, apesar de ter sido descoberta em 1868, ainda apresenta poucos avanços a respeito do tratamento, visto que não há cura, apenas controle da progressão da distrofia. Dessa forma, ainda há muito o que se discutir sobre o manejo adequado desses pacientes a fim de melhorar a qualidade de vida dos mesmos e de seus familiares. Deve-se ressaltar a importância da abordagem multidisciplinar, visto que a fraqueza muscular progressiva e o seu manejo apresentam efeitos que atingem vários sistemas do corpo humano. Assim, o cuidado ao paciente e sua família deve envolver uma série de profissionais: neurologista, pneumologista, cardiologista, ortopedista (em caso de fraturas), endocrinologista, nutricionista, fisioterapeutas e psicólogos.

No caso relatado, o menor apresentava baixa estatura com previsão de estatura final no limite inferior do alvo familiar e deficiência de vitamina $\mathrm{D}$, sendo o indicado a reposição da mesma, a fim de prevenir osteoporose, bem como a monitorização do crescimento e do desenvolvimento puberal. No entanto, como foi visto, esse paciente poderá ter problemas futuros, devendo manter acompanhamento periódico com especialistas. O acompanhamento endocrinológico se torna importante devido aos efeitos da distrofia e do uso de corticosteroides, havendo risco de crise adrenal e diversos efeitos colaterais. No entanto, faltam evidências a respeito dos verdadeiros efeitos endocrinológicos na DMD, mas o acompanhamento se torna essencial pelo risco de retardo no crescimento e puberdade, obesidade e osteoporose.

Futuros estudos podem abordar mais pacientes com Distrofia Muscular de Duchenne, de forma a realizar um trabalho avaliando a associação dos efeitos endocrinológicos com base na dosagem de glicocorticoides utilizada. Além disso, pode ser estudado qual dose apresenta efeito terapêutico necessário associado com menos efeitos colaterais endocrinológicos.

\section{Referências}

Annexstad, E. J., Lund-Petersen, I., \& Rasmussen, M. (2014). Duchenne muscular dystrophy. Tidsskrift for Den Norske Legeforening, 134(14), 1361-1364. https://doi.org/10.4045/tidsskr.13.0836

Araujo, A. P. Q. C., Nardes, F., Fortes, C. P. D. D., Pereira, J. A., Rebel, M. F., Dias, C. M., Barbosa, R. de C. G. A., Lopes, M. V. R., Langer, A. L., Neves, F. R., \& Reis, E. F. (2018). Brazilian consensus on duchenne muscular dystrophy. Part 2: Rehabilitation and systemic care. Arquivos de Neuro-Psiquiatria, 76(7), 481-489. https://doi.org/10.1590/0004-282x20180062

Bianchi, M. L., Morandi, L., Andreucci, E., Vai, S., Frasunkiewicz, J., \& Cottafava, R. (2011a). Low bone density and bone metabolism alterations in Duchenne muscular dystrophy: response to calcium and vitamin D treatment. Osteoporosis International, 22(2), 529-539. https://doi.org/10.1007/s00198-010$1275-5$

Bianchi, M. L., Biggar, D., Bushby, K., Rogol, A. D., Rutter, M. M., \& Tseng, B. (2011b). Endocrine Aspects of Duchenne Muscular Dystrophy. Neuromuscular Disorders, 21(4), 298-303. https://doi.org/10.1016/j.nmd.2011.02.006

Birnkrant, D. J., Bushby, K., Bann, C. M., Apkon, S. D., Blackwell, A., Brumbaugh, D., Case, L. E., Clemens, P. R., Hadjiyannakis, S., Pandya, S., Street, N., Tomezsko, J., Wagner, K. R., Ward, L. M., \& Weber, D. R. (2018). Diagnosis and management of Duchenne muscular dystrophy, part 1: diagnosis, and neuromuscular, rehabilitation, endocrine, and gastrointestinal and nutritional management. The Lancet Neurology, 17(3), 251-267. https://doi.org/10.1016/S1474-4422(18)30024-3 
Research, Society and Development, v. 10, n. 8, e54910817773, 2021

(CC BY 4.0) | ISSN 2525-3409 | DOI: http://dx.doi.org/10.33448/rsd-v10i8.17773

Bushby, K., Finkel, R., Birnkrant, D. J., Case, L. E., Clemens, P. R., Cripe, L., Kaul, A., Kinnett, K., McDonald, C., Pandya, S., Poysky, J., Shapiro, F., Tomezsko, J., \& Constantin, C. (2010). Diagnosis and management of Duchenne muscular dystrophy, part 1: diagnosis, and pharmacological and psychosocial management. The Lancet Neurology, 9(1), 77-93. https://doi.org/10.1016/S1474-4422(09)70271-6

Hoffman, E. P., Brown, R. H., \& Kunkel, L. M. (1987). Dystrophin: The protein product of the duchenne muscular dystrophy locus. Cell, 51(6), 919-928. https://doi.org/10.1016/0092-8674(87)90579-4

Kunkel, L. M. (1986). Analysis of deletions in DNA from patients with Becker and Duchenne muscular dystrophy. Nature, 322(6074), 73-77. https://doi.org/10.1038/322073a0

Leung, D. G., Germain-Lee, E. L., Denger, B. E., \& Wagner, K. R. (2011). Report on the second endocrine aspects of duchenne muscular dystrophy conference December 1-2, 2010, Baltimore, Maryland, USA. Neuromuscular Disorders, 21(8), 594-601. https://doi.org/10.1016/j.nmd.2011.04.008

Moat, S. J., Bradley, D. M., Salmon, R., Clarke, A., \& Hartley, L. (2013). Newborn bloodspot screening for Duchenne Muscular Dystrophy: 21 years experience in Wales (UK). European Journal of Human Genetics, 21(10), 1049-1053. https://doi.org/10.1038/ejhg.2012.301

Parente, R. C. M., Oliveira, M. A. P. De, \& Celeste, R. K. (2012). Relatos e Série de Casos na Era da Medicina Baseada em Evidência. Brazilian Journal Os Videoendoscopic Surgery, 3, 67-70. http://www.sobracil.org.br/revista/jv030302/bjvs030302_063B.pdf

Pereira A. S. et al. (2018). Metodologia da pesquisa científica. UFSM.

Salera, S., Menni, F., Moggio, M., Guez, S., Sciacco, M., \& Esposito, S. (2017). Nutritional Challenges in Duchenne Muscular Dystrophy. Nutrients, 9(6), 594. https://doi.org/10.3390/nu9060594

Suthar, R., \& Sankhyan, N. (2018). Duchenne Muscular Dystrophy: A Practice Update. The Indian Journal of Pediatrics, 85(4), 276-281. https://doi.org/10.1007/s12098-017-2397-y

Ward, L. M., Hadjiyannakis, S., McMillan, H. J., Noritz, G., \& Weber, D. R. (2018). Bone Health and Osteoporosis Management of the Patient With Duchenne Muscular Dystrophy. Pediatrics, 142(Supplement 2), S34-S42. https://doi.org/10.1542/peds.2018-0333E

Ward, L. M., \& Weber, D. R. (2019). Growth, pubertal development, and skeletal health in boys with Duchenne Muscular Dystrophy. Current Opinion in Endocrinology \& Diabetes and Obesity, 26(1), 39-48. https://doi.org/10.1097/MED.0000000000000456

Weber, D. R., Hadjiyannakis, S., McMillan, H. J., Noritz, G., \& Ward, L. M. (2018a). Obesity and endocrine management of the patient with Duchenne muscular dystrophy. Pediatrics, 142(October 2018), S43-S52. https://doi.org/10.1542/peds.2018-0333F

Weber, D. R., Thomas, S., Erickson, S. W., Fox, D., Oleszek, J., Pandya, S., Venkatesh, Y., Westfield, C., \& Ciafaloni, E. (2018b). Bone Health and Endocrine Care of Boys with Duchenne Muscular Dystrophy: Data from the MD STARnet. Journal of Neuromuscular Diseases, 5(4), 497-507. https://doi.org/10.3233/JND-180317

Werneck, L. C., Lorenzoni, P. J., Ducci, R. D. P., Fustes, O. H., Kay, C. S. K., \& Scola, R. H. (2019). Duchenne muscular dystrophy: An historical treatment review. Arquivos de Neuro-Psiquiatria, 77(8), 579-589. https://doi.org/10.1590/0004-282X20190088

Wood, C. L., Straub, V., Guglieri, M., Bushby, K., \& Cheetham, T. (2016). Short stature and pubertal delay in Duchenne muscular dystrophy. Archives of Disease in Childhood, 101(1), 101-106. https://doi.org/10.1136/archdischild-2015-308654

Yin, R. K. (2015). O estudo de caso. Bookman

Yiu, E. M., \& Kornberg, A. J. (2015). Duchenne muscular dystrophy. Journal of Paediatrics and Child Health, 51(8), 759-764 https://doi.org/10.1111/jpc.12868 